\title{
Impaired Insulin Profiles Following a Single Night of Sleep Restriction: The Impact of Acute Sprint Interval Exercise
}

\author{
Emma L. Sweeney, Daniel J. Peart, Irene Kyza, Thomas Harkes, Jason G. Ellis, and lan H. Walshe \\ Northumbria University
}

\begin{abstract}
Experimental sleep restriction (SR) has demonstrated reduced insulin sensitivity in healthy individuals. Exercise is well-known to be beneficial for metabolic health. A single bout of exercise has the capacity to increase insulin sensitivity for up to 2 days. Therefore, the current study aimed to determine if sprint interval exercise could attenuate the impairment in insulin sensitivity after one night of SR in healthy males. Nineteen males were recruited for this randomized crossover study which consisted of four conditions-control, SR, control plus exercise, and sleep restriction plus exercise. Time in bed was $8 \mathrm{hr}(2300-0700)$ in the control conditions and $4 \mathrm{hr}(0300-0700)$ in the SR conditions. Conditions were separated by a 1-week entraining period. Participants slept at home, and compliance was assessed using wrist actigraphy. Following the night of experimental sleep, participants either conducted sprint interval exercise or rested for the equivalent duration. An oral glucose tolerance test was then conducted. Blood samples were obtained at regular intervals for measurement of glucose and insulin. Insulin concentrations were higher in SR than control $(p=.022)$. Late-phase insulin area under the curve was significantly lower in sleep restriction plus exercise than SR $(862 \pm 589$ and $1,267 \pm 558 ; p=.004)$. Glucose area under the curve was not different between conditions $(p=.207)$. These findings suggest that exercise improves the late postprandial response following a single night of SR.
\end{abstract}

Keywords: glucose metabolism, high-intensity exercise, sleep loss

Short sleep durations are becoming increasingly common, with almost $75 \%$ of adults in Great Britain sleeping outside the recommended 7-9 hr each night (Hirshkowitz et al., 2015). Chronic short sleep is associated with increased risk of developing many diseases, including type 2 diabetes (Yaggi et al., 2006). Experimental studies demonstrate impaired glucose control following sleep restriction (SR; Knutson et al., 2007), with a single night of restriction reducing whole-body insulin sensitivity by $20 \%$ (Donga et al., 2010).

The proposed mechanisms underlying impaired glucose regulation following SR include altered peripheral insulin sensitivity (Broussard et al., 2012; Rao et al., 2015) and changes to the metabolic profile, favoring fatty acid transportation (Davies et al., 2014) which may interfere with insulin signaling. However, although the detrimental effects of SR are clear, few studies have focused on strategies to counter the impairment.

Exercise may have the potential to alter these proposed underlying mechanisms (Saner et al., 2018). A single bout of exercise positively impacts glucose regulation for up to $24 \mathrm{hr}$ (Koopman et al., 2005). This improvement in glucose regulation is apparent with various types of exercise (Breen et al., 2011; Gillen et al., 2012), although high-intensity exercise may to be superior to moderate-intensity exercise for improving insulin sensitivity (Ortega et al., 2015; Rynders et al., 2014). Sprint interval exercise has been shown to produce improvements in insulin area under the curve (AUC) and the insulin sensitivity

Sweeney, Peart, Kyza, Harkes, and Walshe are with the Department of Sport, Exercise and Rehabilitation, Faculty of Health and Life Sciences, Northumbria University, Newcastle Upon Tyne, United Kingdom. Ellis is with Northumbria Sleep Research Laboratory, Faculty of Health and Life Sciences, Northumbria University, Newcastle Upon Tyne, United Kingdom. Sweeney (emma.sweeney@northumbria. ac.uk) is corresponding author. index when measured 30-min postcessation of the exercise bout in healthy males (Ortega et al., 2015). Whether or not improvements such as these would occur in sleep-restricted individuals remains unknown.

Exercise appears to be a promising intervention to alleviate the impairment in insulin sensitivity following total sleep deprivation. Two weeks of high-intensity exercise training has been shown to attenuate the insulin response to an oral glucose tolerance test after one night of total sleep deprivation (de Souza et al., 2017). However, it remains unclear if a single bout of exercise performed after partial SR can produce similar outcomes.

Consequently, this study aimed to investigate the effect of a single bout of sprint interval exercise on whole-body insulin sensitivity, following a single night of SR. We hypothesized that insulin sensitivity would be reduced following SR and that this effect would be attenuated when exercise was performed.

\section{Methods}

\section{Participants}

Nineteen healthy males (mean $\pm S D$; age $25 \pm 8$ years, body mass $81.4 \pm 12.0 \mathrm{~kg}$, stature $180 \pm 7 \mathrm{~cm}$ ) participated in this study. Exclusion criteria included shift workers; regular travel across time zones ( $>3$ times a year) or in the past 4 weeks; presence of any disorders which may influence glycemic control (such as diabetes) or sleep (such as obstructive sleep apnea); current or previous medication in the past year which may have impacted on glucose metabolism or sleep; alteration of sleep, dietary, or physical activity patterns in the previous 3 months; a history of drug or alcohol abuse or eating disorders; following a specific diet which may influence the results, such as intermittent fasting; a habitual bedtime before $2200 \mathrm{hr}$ or after $0100 \mathrm{hr}$; or a habitual 
wake time before $0600 \mathrm{hr}$ or after $0900 \mathrm{hr}$. Individuals were also excluded if they had a contraindication to exercise; poor sleep quality (defined as a Pittsburgh Sleep Quality Index PSQI, score of above 5; Buysse et al., 1989); or were classed as extreme morning or evening types, assessed by the MorningnessEveningness Questionnaire (Horne \& Ostberg, 1976). The study protocol was approved through the Northumbria University ethical approval system. All participants provided written informed consent prior to participation.

\section{Study Design}

This randomized crossover trial consisted of a familiarization visit and 4 one-night experimental trials. The experimental conditions were control (CON), control plus exercise (CE), SR, and sleep restriction plus exercise (SRE). Each condition involved a single night of either 8-hr time in bed from 2300 to $0700 \mathrm{hr}$ (CON and CE) or 4-hr time in bed from 0300 to $0700 \mathrm{hr}$ (SR and SRE). Four-hour sleep has been shown to reduce insulin sensitivity in previous research (Donga et al., 2010). The morning following the control or restricted sleep, participants either rested (CON and SR) or undertook a bout of sprint interval exercise (CE and SRE). All experimental conditions were separated by at least 1 week to prevent carryover effects (van Leeuwen et al., 2010), but no more than 3 weeks.

Throughout the study, participants slept at home. Experimental trials were preceded by a 1-week entraining period, in which participants were asked to keep a consistent bed and wake time. Wrist actigraphy (GeneActiv; Activinsights Ltd, United Kingdom) was used in conjunction with time-stamped text messages to ensure compliance. Participants sent hourly messages to the researcher between 2300 and $0300 \mathrm{hr}$ in SR and SRE conditions. Participants were informed in which condition they were on the day before each laboratory visit.

Diet was provided for the day preceding each experimental trial and was replicated across conditions. Diet was individualized from food diaries issued during the familiarization session, and total energy, carbohydrates, fat, and protein were kept within $10 \%$ of habitual intake. Participants were instructed to eat only the foods provided and to avoid consumption of any caffeine or alcohol, however, were permitted to drink water ad libitum. Participants were also asked to refrain from exercise and napping during this time.

\section{Study Protocol}

Familiarization. One week prior to the first experimental visit, participants attended the laboratory for a screening and familiarization visit. Upon arrival, participants were briefed on the study protocol and given the opportunity to ask questions before completing written informed consent. They completed several screening questionnaires - a physical activity readiness questionnaire, the Pittsburgh Sleep Quality Index (Buysse et al., 1989), and the Morningness-Eveningness Questionnaire (Horne \& Ostberg, 1976). Following satisfactory completion of the screening questionnaires, they were issued with a 3-day food diary, actigraphy watch, and 7-day sleep diary.

Body mass and stature were measured using balance scales (Seca, Birmingham, United Kingdom) and a free-standing stadiometer (Seca). Stature was measured to the nearest $0.1 \mathrm{~cm}$ and body mass to the nearest $0.1 \mathrm{~kg}$.

Participants then underwent an exercise familiarization, which was a reduced version of the study exercise protocol. The familiarization exercise bout consisted of a 5-min warm-up at $70 \mathrm{~W}$ on a cycle ergometer (Monark, Vansbro, Sweden), followed by two all-out 30 -s sprints against $7.5 \%$ of their body mass. Sprints were separated by $4.5 \mathrm{~min}$ of active recovery at a self-selected pace. A 5-min cooldown was then completed.

Experimental Trials. Participants went to bed at $2300 \mathrm{hr}$ or 0300 $\mathrm{hr}$, depending on the condition. After getting up at $0700 \mathrm{hr}$, participants arrived at the laboratory by public transport in a rested state at $0800 \mathrm{hr}$, following a 10 -hr overnight fast. Upon arrival, participants either completed a bout of sprint interval exercise (CE and SRE) or rested in a seated position for the equivalent duration (CON and SR). In all conditions, participants were given a 30-min recovery period, resting in a seated position. After the recovery period, a 2-hr oral glucose tolerance test (OGTT) was conducted, and blood samples were drawn at regular intervals. The OGTT consisted of 82.5-g dextrose (MyProtein, Northwich, United Kingdom) mixed with 300-ml water, consumed within 5 min. During the OGTT, participants remained seated in the laboratory but were permitted to complete sedentary tasks. Participants left the laboratory at approximately $1130 \mathrm{hr}$.

Exercise. The exercise protocol used in this study was based on previous research by Ortega et al. (2015), who demonstrated a $142 \%$ increase in intravenous glucose tolerance test (IVGTT)derived insulin sensitivity measured $30 \mathrm{~min}$ following sprint interval exercise in healthy males. All exercise was performed on a cycle ergometer (Monark Ergomedic 894E; Monark), preceded and proceeded with a 5-min warm-up and cooldown at $70 \mathrm{~W}$.

The sprint interval exercise consisted of four all-out 30 -s sprints against $7.5 \%$ of body mass, interspersed with $4.5 \mathrm{~min}$ of recovery. During the active recovery period, participants cycled at a self-selected pace against a resistance of $1 \mathrm{~kg}$, which was the lowest permitted by the ergometer. Verbal encouragement was given throughout each sprint by the same researcher.

Blood Collection and Processing. Blood was collected at baseline (0), 15, 30, 45, 60, 90, and 120 min during the OGTT using the cannulation technique. For each sample, $8 \mathrm{ml}$ was drawn into a syringe and transferred to a $10-\mathrm{ml}$ serum vacutainer (Becton Dickinson, Stockholm, Sweden). Vacutainers were inverted to ensure thorough mixing. The samples were left to clot at room temperature for $30 \mathrm{~min}$ before being centrifuged at 3,500 rpm at $4{ }^{\circ} \mathrm{C}$ for $15 \mathrm{~min}$. Serum was aliquoted into microtubes. A small amount of serum from each sample was taken up into a capillary tube and placed in microtubes containing $1 \mathrm{ml}$ of hemolyzing solution (EKF Diagnostics, Cardiff, United Kingdom) for determination of glucose concentration. The remaining serum was frozen at $-80{ }^{\circ} \mathrm{C}$ until further analysis.

Serum glucose was measured using the Biosen C-Line automatic glucose analyzer (EKF Diagnostics, Barleben, Germany). Serum insulin was measured using commercially available ELISA kits (Mercodia, Uppsala, Sweden), conducted according to manufacturer's instructions. Intraassay and interassay coefficients of variation were $6 \%$ and $14 \%$, respectively.

\section{Data Analysis}

Sample Size Calculation. Sample size was calculated using G*Power (version 3.1.9.2; Düsseldorf University, Düsseldorf, Germany; Faul et al., 2007). Based on previous work showing a difference of $26.9 \mathrm{mg} / \mathrm{dl}$ in 2-hr glucose values during an OGTT between control and exercise conditions (Rynders et al., 2014), 18 participants were required to achieve $90 \%$ power. 


\section{Statistical Analysis}

Data are presented as mean $\pm S D$. AUC for glucose and insulin were calculated using the trapezoidal rule. AUC was calculated for total ( $2 \mathrm{hr}$ ), early phase (0-60 $\mathrm{min})$, and late phase (60-120 min) during the OGTT. Homeostatic model assessment of insulin resistance (HOMA-IR) (Matthews et al., 1985) and Matsuda index (Matsuda \& DeFronzo, 1999) were calculated to estimate insulin resistance and whole-body insulin sensitivity.

Data were analyzed using SPSS Statistics (version 22; IBM, Portsmouth, United Kingdom). Shapiro-Wilk tests were used to check for normality, and any data which violated the assumption of normality were transformed using a natural logarithm transformation. Linear mixed modeling was used to compare glucose and insulin between conditions. The $p<.05$ was used to indicate significance.

\section{Results}

\section{Sleep}

Average time to bed, wake time, time in bed, and total sleep time in each condition are presented in Table 1. Differences in total sleep time were observed between CON and SR (mean difference $142 \mathrm{~min}$; $p<.001$ ), CE and SRE (mean difference $182 \mathrm{~min} ; p<.001$ ), CE and SRE (mean difference $181 \mathrm{~min} ; p<.001$ ), and CON and SRE (mean difference $141 \mathrm{~min} ; p<.001)$. No differences were observed between $\mathrm{CON}$ and $\mathrm{CE}(p=.581)$ or SR and $\operatorname{SRE}(p=1.000)$.

\section{Exercise}

Peak power output during each of the 30-s sprints is outlined in Table 2. Peak power output did not differ between conditions $(p=.644)$, but a difference was observed over time $(p<.001)$, with

\section{Table 1 Sleep Variables for Each Experimental Condition}

\begin{tabular}{lcccc}
\hline & CON & CE & SR & SRE \\
\hline Bedtime (hhmm) & 2303 & 2306 & 0305 & 0255 \\
Wake time (hhmm) & 0658 & 0659 & 0700 & 0657 \\
TIB (min) & $472 \pm 27$ & $472 \pm 22$ & $236 \pm 19$ & $244 \pm 15$ \\
TST (min) & $337 \pm 95$ & $377 \pm 61$ & $195 \pm 43^{* * * * *}$ & $196 \pm 37 * * *$ \\
\hline
\end{tabular}

Note. Data are mean $\pm S D$. TIB $=$ time in bed; TST $=$ total sleep time; $\mathrm{CON}=$ control; $\mathrm{CE}=$ control plus exercise; $\mathrm{SR}=$ sleep restriction; $\mathrm{SRE}=$ sleep restriction plus exercise.

*Difference from CON $(p<.05)$. **Difference from CE $(p<.05)$. peak power output significantly higher in the first sprint compared with the third (mean difference $106 \mathrm{~W} ; p=.009$ ) and fourth (mean difference $118 \mathrm{~W} ; p=.007)$, and higher in the second compared with the fourth (mean difference $60 \mathrm{~W} ; p=.041$ ). Total work done was similar between conditions $(63,666 \pm 12,029 \mathrm{~J}$ in $\mathrm{CE}$ and $65,045 \pm 11,294 \mathrm{~J}$ in SRE; $p=.340)$.

\section{Glucose and Insulin}

Glucose and insulin concentrations during the OGTT are displayed in Figure 1. Total, early phase, and late-phase AUC for glucose and insulin are shown in Figure 2.

Glucose concentrations did not show evidence of an effect of condition $(p=.216)$ or interaction effect $(p=.146)$. However, there was a significant effect of time during the OGTT $(p<.001)$. No significant difference between conditions was found for peak glucose $(p=.158)$. There was no evidence of an effect of condition $(p=.207)$ for total glucose AUC. Likewise, late- and early phase glucose AUC did not show any significant differences between conditions ( $p=.264$ and $p=.122$, respectively).

Insulin concentrations demonstrated an overall difference between conditions $(p=.019)$, time points $(p<.001)$, and an interaction between conditions and time points $(p=.014)$. Post hoc analyses on these overall differences revealed significantly higher insulin concentrations in SR compared with $\mathrm{CON}$ ( $p=$ .022), with SR showing higher concentrations than CON at $30 \mathrm{~min}(40.04 \pm 23.17$ and $25.79 \pm 13.94 \mu \mathrm{IU} / \mathrm{ml} ; p=.004)$. Insulin concentrations were higher in SR compared with SRE at $60 \mathrm{~min}$ $(31.40 \pm 13.99$ and $21.03 \pm 10.67 \mu \mathrm{IU} / \mathrm{ml} ; p=.042), 90 \mathrm{~min}(19.47 \pm$ 11.11 and $15.52 \pm 15.63 \mu \mathrm{IU} / \mathrm{ml} ; p=.002)$, and $120 \mathrm{~min}(14.12 \pm$ 14.84 and $5.99 \pm 5.27 \mu \mathrm{IU} / \mathrm{ml} ; p=.003)$. Total insulin AUC displayed a trend for an effect of condition $(p=.075)$, with SR tending to be higher than CON $(p=.064)$. Early phase and late-phase insulin AUC also showed significant main effects of condition $(p=.010$ and $p<.001$, respectively). The early phase insulin AUC was higher in SR than CON $(1,472 \pm 811$ and 2,044 $\pm 1,129 ; p=.048)$. Late phase displayed a lower AUC in SRE than SR $(1,267 \pm 558$ and $862 \pm 589$; $p=.004)$.

The HOMA-IR showed a main effect of condition $(p=.019)$, with SR higher than CON $(0.87 \pm 0.99$ and $1.64 \pm 2.60 ; p=.029)$. Matsuda index was significantly different between conditions $(p=.003)$, with SR displaying a lower index than CON (25.31 \pm 20.80 and $12.11 \pm 6.38 ; p=.020)$.

\section{Discussion}

The present study demonstrated that one night of SR impaired insulin sensitivity and that performing sprint interval exercise after SR may improve the late postprandial response.

Table 2 Peak Power Output and Total Work Done During Sprints

\begin{tabular}{lcccc}
\hline & \multicolumn{2}{c}{ PPO (W) } & \multicolumn{2}{c}{ Total work (J) } \\
\cline { 2 - 3 } & CE & SRE & CE & SRE \\
\hline 1 & $857 \pm 189$ & $853 \pm 187$ & $18,356 \pm 2,903$ & $18,355 \pm 3,471$ \\
2 & $818 \pm 177$ & $804 \pm 160$ & $16,551 \pm 2,921$ & $16,580 \pm 3,139$ \\
3 & $762 \pm 194$ & $736 \pm 172$ & $15,488 \pm 2,842$ & $15,424 \pm 3,237$ \\
4 & $733 \pm 179$ & $746 \pm 144$ & $14,686 \pm 2,408$ & $15,502 \pm 2,490$ \\
\hline \multicolumn{2}{l}{ Note. PPO and total work during four all-out 30-s sprints performed in the morning after a night of $8 \mathrm{hr}(\mathrm{CE})$ or $4 \mathrm{hr}$ (SRE) } \\
time in bed. No significant differences were observed between conditions. Data are presented as mean $\pm S D$. CE = control \\
\multicolumn{2}{l}{ plus exercise; SRE = sleep restriction plus exercise; PPO = peak power output. }
\end{tabular}



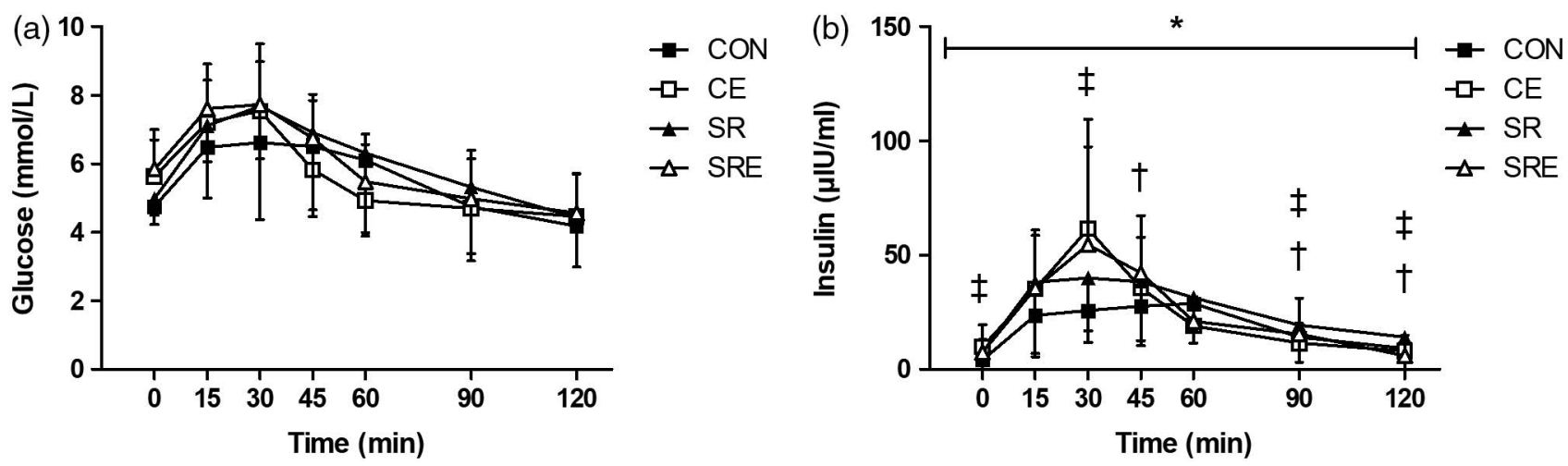

Figure 1 - Glucose (a) and insulin (b) concentrations during the OGTT. OGTT = oral glucose tolerance test; $\mathrm{CON}=\mathrm{control}$; $\mathrm{SR}=$ sleep restriction; $\mathrm{CE}=$ control plus exercise; $\mathrm{SRE}=$ sleep restriction plus exercise. $\mathrm{p}<.05$ indicates significance. *Main effect of condition, with $\mathrm{SR}$ higher than $\mathrm{CON}$. $\ddagger$ Difference between CON and SR. $\dagger$ Difference between SR and SRE.
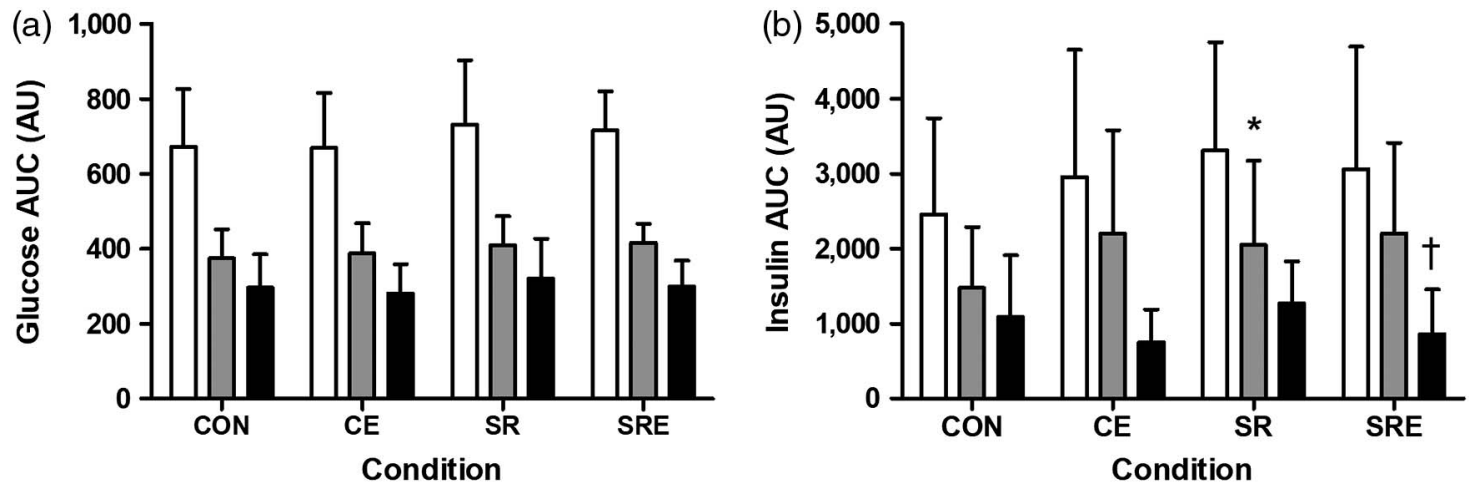

Figure 2 - Total (white bars), early phase (gray shaded bars), and late-phase (black bars) AUC for glucose (a) and insulin (b). Data are presented as mean \pm SEM. Main effect of condition for early and late AUC. * Significant difference $(p<.05)$ compared with CON in early phase. ${ }^{\dagger}$ Significant difference $(p<.05)$ compared with $\mathrm{SR}$ in late phase. $\mathrm{CON}=$ control; $\mathrm{SR}=$ sleep restriction; $\mathrm{CE}=$ control plus exercise; $\mathrm{SRE}=$ sleep restriction plus exercise; AUC $=$ area under the curve.

Participants displayed reduced insulin sensitivity after SR, indicated by increased insulin concentrations during the OGTT and decreased Matsuda index in SR compared with CON. This is consistent with previous research that has demonstrated reduced whole-body insulin sensitivity after a single night (Donga et al., 2010) and multiple nights of SR (Sweeney et al., 2017; Wang et al., 2016). SR may impair whole-body insulin sensitivity through alteration of peripheral insulin signaling, with five nights of SR reducing peripheral, but not hepatic, insulin sensitivity (Rao et al., 2015). Furthermore, SR reduces Akt phosphorylation, which plays a key role in the insulin signaling pathway in peripheral tissues (Broussard et al., 2012).

We hypothesized that a bout of sprint interval exercise would attenuate the impairment in insulin sensitivity in sleep-restricted individuals. Although total insulin AUC was not significantly altered after the exercise bout, our findings suggest that there was an alteration to the late postprandial response when comparing the sleep restricted conditions. However, we did not observe an improvement in insulin or glucose profiles after exercise in the early phase of the OGTT. Our findings are in contrast to Ortega et al. (2015), who demonstrated improved insulin and glucose $60 \mathrm{~min}$ following high-intensity exercise. Methodological differences may explain this discrepancy, as an IVGTT was employed by
Ortega et al., whereas we used an OGTT. Gastric emptying plays a role during the OGTT, whereas this is bypassed when glucose is injected rather than ingested orally. Gastric emptying rate may be slowed by intermittent high-intensity exercise (Leiper et al., 2001), delaying the absorption of the glucose drink, and therefore, findings may not be comparable between an OGTT and IVGTT. Alternatively, it may be possible that SR alters the metabolic response to exercise.

Our findings reflect those by Rynders et al. (2014), who noted improvements in the late rather than total postprandial response after a bout of exercise. High-intensity exercise is known to temporarily increase glucose due to gluconeogenesis and possible carbohydrate sparing for glycogen repletion (Marliss et al., 1992). It may be possible that as glucose regulation was measured $30 \mathrm{~min}$ after cessation of exercise, the temporary alterations in glucose regulation which occur during exercise were still influencing our measurements. In addition, although not statistically significant, it appears that insulin concentrations in the early phase of the OGTT were increased after exercise compared with rest in the current study. An increase in early phase insulin secretion may influence late-phase responses, potentially through suppression of endogenous glucose production (Del Prato et al., 2002). This early phase response may therefore contribute to the lower late-phase insulin 
AUC which was observed in SRE compared with SR. The late phase of the OGTT has been shown to predict incident diabetes independent of the early phase (Lorenzo et al., 2012), suggesting that despite no overall change, a decrease in late-phase insulin AUC may be beneficial.

Although the present study suggests that there is potential for exercise to positively influence the postprandial response to an OGTT in sleep-restricted individuals, it was not designed to identify the possible mechanisms. Previous research has suggested that insulin action is improved after exercise through altered phosphorylation of components of the insulin signaling pathway in peripheral tissues (Wojtaszewski \& Richter, 2006). As SR is thought to negatively impact peripheral insulin sensitivity (Broussard et al., 2012; Rao et al., 2015), it can be speculated that exercise improves insulin sensitivity in sleep-restricted individuals through alteration of insulin action in peripheral tissues.

The present study has some limitations which should be noted. First, the study population consisted only of healthy males, meaning it may not be feasible to extrapolate the findings to other populations including females and individuals with metabolic abnormalities. Second, an OGTT does not enable measurement of metabolic characteristics, such glucose disposal and uptake, so gives limited information regarding the decrease in insulin sensitivity.

In conclusion, sprint interval exercise may offer some potential to attenuate the impairments in insulin sensitivity following a single night of reduced sleep. This may have implications for individuals facing sleep curtailment. To our knowledge, this is the first study to explore the effect of acute exercise on insulin sensitivity following SR. Therefore, future research may investigate whether exercise modality, intensity, duration, or timing influences the change in glucose regulation in sleep-restricted individuals. It would also be beneficial to investigate the time course of improved glucose regulation to determine whether the benefit of exercise persists for multiple hours as is the case in nonsleep-restricted individuals.

\section{Acknowledgments}

The authors would like to thank the study participants for their time and commitment to the study. They would also like to thank Gino Gamboa for assisting with data collection. The study was designed by E.L. Sweeney, I.H. Walshe, D.J. Peart, and J.G. Ellis; data were collected and analyzed by E.L. Sweeney, I. Kyza, T. Harkes, and I.H. Walshe; data interpretation and preparation of manuscript were conducted by E.L. Sweeney, I.H. Walshe, and D.J. Peart; all authors provided comments on the manuscript and approved the final version. The authors have no conflicts of interest to declare.

\section{References}

Breen, L., Philp, A., Shaw, C.S., Jeukendrup, A.E., Baar, K., \& Tipton, K.D. (2011). Beneficial effects of resistance exercise on glycemic control are not further improved by protein ingestion. PLoS One, 6(6), e20613. PubMed ID: 21701685 doi:10.1371/journal.pone. 0020613

Broussard, J.L., Ehrmann, D.A., Van Cauter, E., Tasali, E., \& Brady, M.J. (2012). Impaired insulin signaling in human adipocytes after experimental sleep restriction: A randomized, crossover study. Annals of Internal Medicine, 157(8), 549-557. PubMed ID: 23070488 doi:10. 7326/0003-4819-157-8-201210160-00005
Buysse, D.J., Reynolds, C.F., 3rd, Monk, T.H., Berman, S.R., \& Kupfer, D.J. (1989). The Pittsburgh Sleep Quality Index: A new instrument for psychiatric practice and research. Psychiatry Research, 28(2), 193-213. PubMed ID: 2748771 doi:10.1016/0165-1781(89)90047-4

Davies, S.K., Ang, J.E., Revell, V.L., Holmes, B., Mann, A., Robertson, F.P., . . Skene, D.J. (2014). Effect of sleep deprivation on the human metabolome. Proceedings of the National Academy of Sciences, 111(29), 10761. doi:10.1073/pnas.1402663111

de Souza, J.F.T., Dattilo, M., de Mello, M.T., Tufik, S., \& Antunes, H.K.M. (2017). High-intensity interval training attenuates insulin resistance induced by sleep deprivation in healthy males. Frontiers in Physiology, 8, 992. PubMed ID: 29270126 doi:10.3389/fphys.2017. 00992

Del Prato, S., Marchetti, P., \& Bonadonna, R.C. (2002). Phasic insulin release and metabolic regulation in type 2 diabetes. Diabetes, 51(Suppl 1), S109-S116. doi:10.2337/diabetes.51.2007.S109

Donga, E., van Dijk, M., van Dijk, J.G., Biermasz, N.R., Lammers, G.J., van Kralingen, K.W., . . Romijn, J.A. (2010). A single night of partial sleep deprivation induces insulin resistance in multiple metabolic pathways in healthy subjects. The Journal of Clinical Endocrinology and Metabolism, 95(6), 2963-2968. PubMed ID: 20371664 doi:10.1210/jc.2009-2430

Faul, F., Erdfelder, E., Lang, A.-G., \& Buchner, A. (2007). G*Power 3: A flexible statistical power analysis program for the social, behavioral, and biomedical sciences. Behavior Research Methods, 39(2), 175191. PubMed ID: 17695343 doi:10.3758/BF03193146

Gillen, J.B., Little, J.P., Punthakee, Z., Tarnopolsky, M.A., Riddell, M.C., \& Gibala, M.J. (2012). Acute high-intensity interval exercise reduces the postprandial glucose response and prevalence of hyperglycaemia in patients with type 2 diabetes. Diabetes, Obesity and Metabolism, 14(6), 575-577. PubMed ID: 22268455 doi:10.1111/j.1463-1326. 2012.01564.x

Hirshkowitz, M., Whiton, K., Albert, S.M., Alessi, C., Bruni, O., DonCarlos, L., . . . Adams Hillard, P.J. (2015). National Sleep Foundation's sleep time duration recommendations: Methodology and results summary. Sleep Health: Journal of the National Sleep Foundation, 1(1), 40-43. doi:10.1016/j.sleh.2014.12.010

Horne, J.A., \& Ostberg, O. (1976). A self-assessment questionnaire to determine morningness-eveningness in human circadian rhythms. International Journal of Chronobiology, 4(2), 97-110. PubMed ID: 1027738

Knutson, K.L., Spiegel, K., Penev, P., \& Van Cauter, E. (2007). The metabolic consequences of sleep deprivation. Sleep Medicine Reviews, 11(3), 163-178. PubMed ID: 17442599 doi:10.1016/j.smrv. 2007.01.002

Koopman, R., Manders, R.J., Zorenc, A.H., Hul, G.B., Kuipers, H., Keizer, H.A., \& van Loon, L.J. (2005). A single session of resistance exercise enhances insulin sensitivity for at least $24 \mathrm{~h}$ in healthy men. European Journal of Applied Physiology, 94(1-2), 180-187. doi:10. 1007/s00421-004-1307-y

Leiper, J.B., Broad, N.P., \& Maughan, R.J. (2001). Effect of intermittent high-intensity exercise on gastric emptying in man. Medicine \& Science in Sports \& Exercise, 33(8), 1270-1278. doi:10.1097/ 00005768-200108000-00005

Lorenzo, C., Williams, K., \& Haffner, S.M. (2012). Insulin secretion based on the late oral glucose tolerance test period and incident diabetes: The San Antonio Heart Study. Diabetic Medicine: A Journal of the British Diabetic Association, 29(8), e151-e158. doi:10.1111/j.14645491.2012.03660.x

Marliss, E.B., Simantirakis, E., Miles, P.D., Hunt, R., Gougeon, R., Purdon, C., ... Vranic, M. (1992). Glucose turnover and its regulation during intense exercise and recovery in normal male subjects. 
Clinical and Investigative Medicine, 15(5), 406-419. PubMed ID: 1458713

Matsuda, M., \& DeFronzo, R.A. (1999). Insulin sensitivity indices obtained from oral glucose tolerance testing: Comparison with the euglycemic insulin clamp. Diabetes Care, 22(9), 1462. PubMed ID: 10480510 doi:10.2337/diacare.22.9.1462

Matthews, D.R., Hosker, J.P., Rudenski, A.S., Naylor, B.A., Treacher, D.F., \& Turner, R.C. (1985). Homeostasis model assessment: Insulin resistance and beta-cell function from fasting plasma glucose and insulin concentrations in man. Diabetologia, 28(7), 412-419. PubMed ID: 3899825 doi:10.1007/BF00280883

Ortega, J.F., Fernández-Elías, V.E., Hamouti, N., Pallares, J.G., \& MoraRodriguez, R. (2015). Higher insulin-sensitizing response after sprint interval compared to continuous exercise. International Journal of Sports Medicine, 36(03), 209-214.

Rao, M. N., Neylan, T.C., Grunfeld, C., Mulligan, K., Schambelan, M., \& Schwarz, J.-M. (2015). Subchronic sleep restriction causes tissuespecific insulin resistance. The Journal of Clinical Endocrinology and Metabolism, 100(4), 1664-1671. PubMed ID: 25658017 doi:10. 1210/jc.2014-3911

Rynders, C.A., Weltman, J.Y., Jiang, B., Breton, M., Patrie, J., Barrett, E.J., \& Weltman, A. (2014). Effects of exercise intensity on postprandial improvement in glucose disposal and insulin sensitivity in prediabetic adults. The Journal of Clinical Endocrinology \& Metabolism, 99(1), 220-228. PubMed ID: 24243632 doi:10.1210/jc.20132687
Saner, N.J., Bishop, D.J., \& Bartlett, J.D. (2018). Is exercise a viable therapeutic intervention to mitigate mitochondrial dysfunction and insulin resistance induced by sleep loss? Sleep Medicine Reviews, 37, 60-68. PubMed ID: 29056415 doi:10.1016/j.smrv.2017.01.001

Sweeney, E.L., Jeromson, S., Hamilton, D.L., Brooks, N.E., \& Walshe, I.H. (2017). Skeletal muscle insulin signaling and whole-body glucose metabolism following acute sleep restriction in healthy males. Physiological Reports, 5(23), e13498. doi:10.14814/phy2. 13498

van Leeuwen, W.M.A., Hublin, C., Sallinen, M., Härmä, M., Hirvonen, A., \& Porkka-Heiskanen, T. (2010). Prolonged sleep restriction affects glucose metabolism in healthy young men. International Journal of Endocrinology, 2010, 108641. PubMed ID: 20414467 doi:10.1155/2010/108641

Wang, X., Greer, J., Porter, R.R., Kaur, K., \& Youngstedt, S.D. (2016). Short-term moderate sleep restriction decreases insulin sensitivity in young healthy adults. Sleep Health in Early Care and Education, 2(1), 63-68. doi:10.1016/j.sleh.2015.11.004

Wojtaszewski, J.F., \& Richter, E.A. (2006). Effects of acute exercise and training on insulin action and sensitivity: Focus on molecular mechanisms in muscle. Essays in Biochemistry, 42, 31-46. PubMed ID: 17144878 doi:10.1042/bse0420031

Yaggi, H.K., Araujo, A.B., \& McKinlay, J.B. (2006). Sleep duration as a risk factor for the development of type 2 diabetes. Diabetes Care, 29(3), 657. PubMed ID: 16505522 doi:10.2337/diacare.29.03.06. dc05-0879 